\title{
Kantowski-Sachs Two Fluid Radiating Cosmological Model in Brans-Dicke Theory of Gravitation
}

\author{
V.U.M.Rao, G. Suryanarayana, Y.Aditya \\ Department of Applied Mathematics, Andhra University, Visakhapatnam, India \\ Email: umrao57@hotmail.com
}

\begin{abstract}
Spatially homogeneous Kantowski-Sachs cosmological model filled with barotropic fluid and dark energy is obtained in a scalar tensor theory of gravitation proposed by Brans and Dicke (1961). We consider both cases when the dark energy is minimally coupled to barotropic fluid as well as directly interacts with it. In these two cases the EoS parameter of dark energy is in good agreement with the observational data. Also some important features of the models, thus obtained, have been discussed.
\end{abstract}

Keywords: Kantowski-Sachs metric, Brans-Dicke theory, two-fluids, dark energy, EoS Parameter

\section{Introduction}

In the study of modern cosmology, we consider that the total energy density of the universe is dominated by the densities of two components: the dark matter and the dark energy. The recent observational data strongly motivate researchers to study general properties of cosmological models containing more than one fluid. These universes are modeled with perfect fluids and with mixtures of non-interacting fluids under the assumption that there is no energy transfer among the components. But, such scenarios are not confirmed by observational data. This motivates us to study cosmological models containing fluids which interact with each other. In recent years there has been immense interest in cosmological models with dark energy in general relativity because of the fact that our observable universes is undergoing a phase of accelerated expansion which has been confirmed by several cosmological observations such as type 1a supernova by several authors (Riess et al. [1], [2], [3] Perlmutter et al.[4], [5], [6], Knop et al. [7]). Caldwell [8] \& Huange [9] have discussed cosmic microwave background (CMB) anisotropy and Daniel et al. [10] have studied large scale structure and strongly indicate that dark energy dominates the present universe, causing cosmic acceleration. Based on these observations, cosmologists have accepted the idea of dark energy, which is a fluid with negative pressure making up around $70 \%$ of the present universe energy content to be responsible for this acceleration due to repulsive gravitation.

Cosmologists have proposed many candidates for dark energy to fit the current observations such as cosmological constant, tachyon, quintessence, phantom and so on. Evolution of the equation of state (EoS) of dark energy $\omega_{D}=p_{D} / \rho_{D}$ has transferred from $\omega_{D}>-1$ in the near past (quintessence region) to $\omega_{D}<-1$ at recent stage (phantom region). Akarsu and Kilinc [11], [12], Yadav [13], Yadav and Yadav [14], Pradhan et al. [15],[16], Pradhan and Amirhashchi [17] and Yadav et al. [18] have investigated different aspects of dark energy models in general relativity with variable EoS parameter. The concept of dark energy was proposed for understanding this currently accelerating expansion of the universe, and then its existence was confirmed by several high precision observational experiments (Bennett et al. [19]; Spergel et al. [20]; Tegmark et al. [21]), especially the Wilkinson Microwave Anisotropy Probe (WMAP) satellite experiment. The WMAP shows that dark energy occupies about $73 \%$ of the energy of the universe, and dark matter about $23 \%$. The usual baryon matter, which can be described by our known particle theory, occupies only about $4 \%$ of the total energy of the universe.

This motivates us to study cosmological models containing fluids which interact with each other. Tolman [22] and Davidson [23] have considered the interaction between dust-like matter and radiation. Cataldo et al. [24] have considered the simplest non-trivial cosmological scenarios for an interacting 
mixture of two cosmic fluids described by power-law scale factors, i.e. the expansion as a power-law in time. Whereas, an interacting and a non-interacting two-fluid scenario for dark energy in an FRW universe with constant deceleration parameter has been described by Pradhan et al. [25]. Adhav et al. [26] have investigated interacting cosmic fluids in LRS Bianchi type-I cosmological model. Saha et al. [27] revisited two-fluid scenario for dark energy models in an FRW universe investigated by Amirhashchi et al. [28]. Adhav et al. [29] have studied Kaluza-Klein interacting cosmic fluid cosmological model. Reddy and Santhi Kumar [30] have discussed two-fluid scenario for dark energy model in a scalar-tensor theory of gravitation. Amirhashchi et al. [31] have studied interacting two-fluid viscous dark energy models in a non-flat universe.

Brans-Dicke [32] theory of gravitation is a natural extension of general relativity which introduces an additional scalar field $\varphi$ besides the metric tensor $g_{i j}$ and dimensionless coupling constant $\omega$. The Brans-Dicke field equations combined with scalar and tensor field are given by

$$
R_{i j}-\frac{1}{2} R g_{i j}=-8 \pi \varphi^{-1} T_{i j}-\omega \varphi^{-2}\left(\varphi_{, i} \varphi_{, j}-\frac{1}{2} g_{i j} \varphi_{, k} \varphi^{, k}\right)-\varphi^{-1}\left(\varphi_{i ; j}-g_{i j} \varphi_{; k}{ }^{k}\right)
$$

and

$$
\varphi_{; k}^{, k}=8 \pi(3+2 \omega)^{-1} T
$$

where $R$ is the scalar curvature, $\omega$ and $n$ are constants, $T_{i j}$ is the stress energy tensor of the matter and comma and semicolon denote partial and covariant differentiation respectively.

Also, we have energy conservation equation

$$
T_{; j}^{i j}=0
$$

This equation is a consequence of the field equations (1) and (2).

Several aspects of Brans-Dicke cosmology have been extensively investigated by many authors. The work of Singh and Rai [33] gives a detailed survey of Brans-Dicke cosmological models. Reddy and Rao [34], Banerjee and Santos [35], Shriram [36], Shriram and Singh [37], Berman et al. [38], Reddy [39], Reddy and Naidu [40], Adhav et al.[41], Rao and Vijaya Santhi [42], [43], [44], Naidu et al.[45], Vidya Sagar et al. [46] and Das \& Abdulla [47] are some of the authors who have investigated several aspects of this theory. Recently, Pawar and Solanke [48] have discussed exact Kantowski-Sachs anisotropic dark energy cosmological models in Brans-Dicke theory of gravitation.

In this paper, we will discuss spatially homogeneous Kantowski-Sachs cosmological model filled with barotropic fluid and dark energy in Brans and Dicke [32] theory of gravitation. The plan of the paper is, in section 2 we discuss metric and energy momentum tensor. In section 3, we obtained solutions for interacting and non-interacting cosmological models. We discussed some other important features of the obtained models in section 4 . The last section contains some conclusions.

\section{$2 \quad$ Metric and Energy Momentum Tensor}

We consider a spatially homogeneous Kantowski - Sachs metric of the form

$$
\mathrm{d} s^{2}=\mathrm{d} t^{2}-A^{2} \mathrm{~d} r^{2}-B^{2}\left(\mathrm{~d} \theta^{2}+\sin ^{2} \theta \mathrm{d} \varphi^{2}\right)
$$

where $A \& B$ are the functions of time $t$ only.

The total energy momentum tensor for two fluids is given by

$$
T_{i j}=\left(\rho_{t o t}+p_{t o t}\right) u_{i} u_{j}-p_{t o t} g_{i j}
$$

where $\rho_{\text {tot }}=\rho_{m}+\rho_{D}$ and $p_{t o t}=p_{m}+p_{D}$. Here $\rho_{m}$ and $p_{m}$ are energy density and pressure of barotropic fluid and $\rho_{D} \& p_{D}$ are energy density and pressure of dark fluid respectively, $u^{i}$ is the fourvelocity of the fluid satisfying the following condition,

$$
g_{i j} u^{i} u^{j}=1
$$

In a co-moving coordinate system, we get

$$
T_{1}^{1}=T_{2}^{2}=T_{3}^{3}=-p_{\text {tot }}, T_{4}^{4}=\rho_{\text {tot }} \text { and } T_{j}^{i}=0 \text { for } i \neq j
$$

where the quantities $\rho_{\text {tot }}$ and $p_{\text {tot }}$ are functions of ' $t$ ' only. 


\section{$3 \quad$ Solutions of Field Equations}

Now with the help of (5)-(7), the field equations (1) and (2) for the metric (4) can be written as

$$
\begin{gathered}
2 \frac{\ddot{B}}{B}+\frac{\dot{B}^{2}}{B^{2}}+\frac{1}{B^{2}}+\frac{\omega \dot{\varphi}^{2}}{2 \varphi^{2}}+\frac{\ddot{\varphi}}{\varphi}+2 \frac{\dot{\varphi} \dot{B}}{\varphi B}=-\frac{8 \pi p_{t o t}}{\varphi} \\
\frac{\ddot{A}}{A}+\frac{\ddot{B}}{B}+\frac{\dot{A} \dot{B}}{A B}+\frac{\omega \dot{\varphi}^{2}}{2 \varphi^{2}}+\frac{\ddot{\varphi}}{\varphi}+\frac{\dot{\varphi}}{\varphi}\left(\frac{\dot{A}}{A}+\frac{\dot{B}}{B}\right)=-\frac{8 \pi p_{t o t}}{\varphi} \\
2 \frac{\dot{A} \dot{B}}{A B}+\frac{\dot{B}^{2}}{B^{2}}+\frac{1}{B^{2}}-\frac{\omega \dot{\varphi}^{2}}{2 \varphi^{2}}+\frac{\dot{\varphi}}{\varphi}\left(\frac{\dot{A}}{A}+2 \frac{\dot{B}}{B}\right)=\frac{8 \pi \rho_{t o t}}{\varphi} \\
\ddot{\varphi}+\dot{\varphi}\left(\frac{\dot{A}}{A}+2 \frac{\dot{B}}{B}\right)=\frac{8 \pi}{(3+2 \omega)}\left(\rho_{t o t}-3 p_{t o t}\right)
\end{gathered}
$$

Also the energy conservation equation leads to

$$
\dot{\rho}_{t o t}+3 H\left(\rho_{t o t}+p_{t o t}\right)=0
$$

Here the over head dot denotes differentiation with respect to $t$.

The equations (8) to (12) is a system of four independent equations with five unknowns $A, B, p_{t o t}, \rho_{\text {tot }}$ and $\varphi$. In order to get a deterministic solution we take the following plausible physical condition, the shear scalar $\sigma$ is proportional to scalar expansion $\theta$, which leads to the linear relationship between the metric potentials $A$ and $B$, i.e.,

$$
B=A^{n} \text {, where } n>1 \text { is a constant. }
$$

Using (13) the above field equations (8) to (11) can be written as

$$
\begin{gathered}
2 \frac{\ddot{B}}{B}+\frac{\dot{B}^{2}}{B^{2}}+\frac{1}{B^{2}}+\frac{\omega \dot{\varphi}^{2}}{2 \varphi^{2}}+\frac{\ddot{\varphi}}{\varphi}+2 \frac{\dot{B} \dot{\varphi}}{B \varphi}=-\frac{8 \pi p_{t o t}}{\varphi} \\
(n+1) \frac{\ddot{B}}{B}+n^{2} \frac{\dot{B}^{2}}{B^{2}}+\frac{\omega \dot{\varphi}^{2}}{2 \varphi^{2}}+\frac{\ddot{\varphi}}{\varphi}+(n+1) \frac{\dot{B} \dot{\varphi}}{B \varphi}=-\frac{8 \pi p_{t o t}}{\varphi} \\
(2 n+1) \frac{\dot{B}^{2}}{B^{2}}+\frac{1}{B^{2}}-\frac{\omega \dot{\varphi}^{2}}{2 \varphi^{2}}+(n+2) \frac{\dot{B} \dot{\varphi}}{B \varphi}=\frac{8 \pi \rho_{t o t}}{\varphi} \\
\ddot{\varphi}+(n+2) \frac{\dot{B} \dot{\varphi}}{B \varphi}=\frac{8 \pi}{(3+2 \omega)}\left(\rho_{t o t}-3 p_{t o t}\right)
\end{gathered}
$$

By considering the radiating model, i.e., for

$$
\rho_{\text {tot }}=3 p_{\text {tot }}
$$

the field equation (17) can be written as

$$
\ddot{\varphi}+(n+2) \frac{\dot{B} \dot{\varphi}}{B \varphi}=0
$$

From equations (14), (15) and (19), we get

$$
\frac{\ddot{B}}{B}+(n+1) \frac{\dot{B}^{2}}{B^{2}}-\frac{1}{B^{2}(n-1)}=\frac{\ddot{\varphi}}{\varphi(n+2)}
$$

From equation (20), we get

$$
\begin{aligned}
& A=\left(\frac{k_{1}}{k_{2}} \sin \left(k_{2} t+k_{3}\right)\right)^{n} \\
& B=\left(\frac{k_{1}}{k_{2}}\right) \sin \left(k_{2} t+k_{3}\right)
\end{aligned}
$$

where $k_{2}^{2}=\frac{m^{2}}{n+2}, \quad k_{1}^{2}=\frac{1}{n^{2}-1},(n \neq 1)$ and $k_{3}$ is an integrating constant. 
Also, the scalar field is given by

$$
\varphi=k_{4} \cos k_{6} t+k_{5} \sin k_{6} t
$$

where $k_{4}, k_{5}$ are integrating constants and $k_{6}=m \sqrt{n+2}$.

Thus the metric (4) can be written as

$$
\mathrm{d} s^{2}=\mathrm{d} t^{2}-\left(\frac{k_{1}}{k_{2}}\right)^{2 n} \sin ^{2 n}\left(k_{2} t+k_{3}\right) \mathrm{d} r^{2}-\left(\frac{k_{1}}{k_{2}}\right)^{2} \sin ^{2}\left(k_{2} t+k_{3}\right)\left(\mathrm{d} \theta^{2}+\sin ^{2} \theta \mathrm{d} \varphi^{2}\right)
$$

The EoS of the barotropic fluid and dark energy are respectively given by

$$
\omega_{m}=\frac{p_{m}}{\rho_{m}} \text { and } \omega_{D}=\frac{p_{D}}{\rho_{D}}
$$

In the following sections we deal with two cases, (i) non-interacting two-fluid model and (ii) interacting two-fluid model.

\section{Non-interacting two-fluid model}

First, we consider that two fluids do not interact with each other. Therefore, the general form of conservation equation (3.5) leads us to write the conservation equation for the dark and barotropic fluid separately as,

$$
\dot{\rho}_{m}+3 H\left(\rho_{m}+p_{m}\right)=0
$$

and

$$
\dot{\rho}_{D}+3 H\left(\rho_{D}+p_{D}\right)=0
$$

Here is, of course, a structural difference between equations (26) and (27). Because equation (26) is in the form of $\omega_{m}$ which is constant and hence equation (26) is integrable. But equation (27) is a function of $\omega_{D}$. Accordingly, $\rho_{D}$ and $p_{D}$ are also functions of $\omega_{D}$. Therefore, we cannot integrate equation (27) as it is a function of $\omega_{D}$ which is an unknown time dependent parameter.

Integrating equation (26) leads to

$$
8 \pi p_{m}=8 \pi \rho_{0}\left[\sin \left(k_{2} t+k_{3}\right)\right]^{-(n+2)\left(1+\omega_{m}\right)}
$$

From equations (16), (21) - (23) and (28), we get

where $k_{4}=\cos k_{7}, k_{5}=\sin k_{7}$.

$$
\begin{aligned}
8 \pi \rho_{D} & =k_{2}^{2}\left\{\left(1+2 n+\frac{1}{k_{1}^{2}}\right) \cot ^{2}\left(k_{2} t+k_{3}\right)+\frac{1}{k_{1}^{2}}\right\} \cos \left(k_{6} t-k_{7}\right) \\
& -k_{6}\left\{(n+2) k_{2} \cot \left(k_{2} t+k_{3}\right)+\frac{\omega}{2} k_{6} \tan \left(k_{6} t-k_{7}\right)\right\} \sin \left(k_{6} t-k_{7}\right) \\
& -8 \pi \rho_{0}\left[\sin \left(k_{2} t+k_{3}\right)\right]^{-(n+2)\left(1+\omega_{m}\right)}
\end{aligned}
$$

From equations (25) and (28), we get

$$
8 \pi p_{m}=8 \pi \rho_{0} \omega_{m}\left[\sin \left(k_{2} t+k_{3}\right)\right]^{-(n+2)\left(1+\omega_{m}\right)}
$$

From equations (15), (21) - (23) and (30), we get

$$
\begin{aligned}
8 \pi p_{D} & =\left\{k_{6}{ }^{2}-k_{2}{ }^{2}\left(n^{2} \cot ^{2}\left(k_{2} t+k_{3}\right)-n-1\right)\right\} \cos \left(k_{6} t-k_{7}\right) \\
& +k_{6}\left\{(n+1) k_{2} \cot \left(k_{2} t+k_{3}\right)-\frac{\omega}{2} k_{6} \tan \left(k_{6} t-k_{7}\right)\right\} \sin \left(k_{6} t-k_{7}\right) \\
& -8 \pi \rho_{0} \omega_{m}\left[\sin \left(k_{2} t+k_{3}\right)\right]^{-(n+2)\left(1+\omega_{m}\right)}
\end{aligned}
$$

From equations (25), (29) and (31), we get 


$$
\begin{aligned}
& \left\{k_{6}^{2}-k_{2}^{2}\left(n^{2} \cot ^{2}\left(k_{2} t+k_{3}\right)-n-1\right)\right\} \cos \left(k_{6} t-k_{7}\right)-8 \pi \rho_{0} \omega_{m}\left[\sin \left(k_{2} t+k_{3}\right)\right]^{-(n+2)\left(1+\omega_{m}\right)} \\
\omega_{D}= & \frac{+k_{6}\left\{(n+1) k_{2} \cot \left(k_{2} t+k_{3}\right)-\frac{\omega}{2} k_{6} \tan \left(k_{6} t-k_{7}\right)\right\} \sin \left(k_{6} t-k_{7}\right)}{k_{2}^{2}\left\{\left(1+2 n+\frac{1}{k_{1}^{2}}\right) \cot ^{2}\left(k_{2} t+k_{3}\right)+\frac{1}{k_{1}^{2}}\right\} \cos \left(k_{6} t-k_{7}\right)-8 \pi \rho_{0}\left[\sin \left(k_{2} t+k_{3}\right)\right]^{-(n+2)\left(1+\omega_{m}\right)}} \\
- & k_{6}\left\{(n+2) k_{2} \cot \left(k_{2} t+k_{3}\right)+\frac{\omega}{2} k_{6} \tan \left(k_{6} t-k_{7}\right)\right\} \sin \left(k_{6} t-k_{7}\right)
\end{aligned}
$$

Thus the metric (24) together with (23) and (28)-(32) constitutes Kantowski-Sachs non-interacting two-fluid cosmological model in Brans-Dicke scalar tensor theory of gravitation.

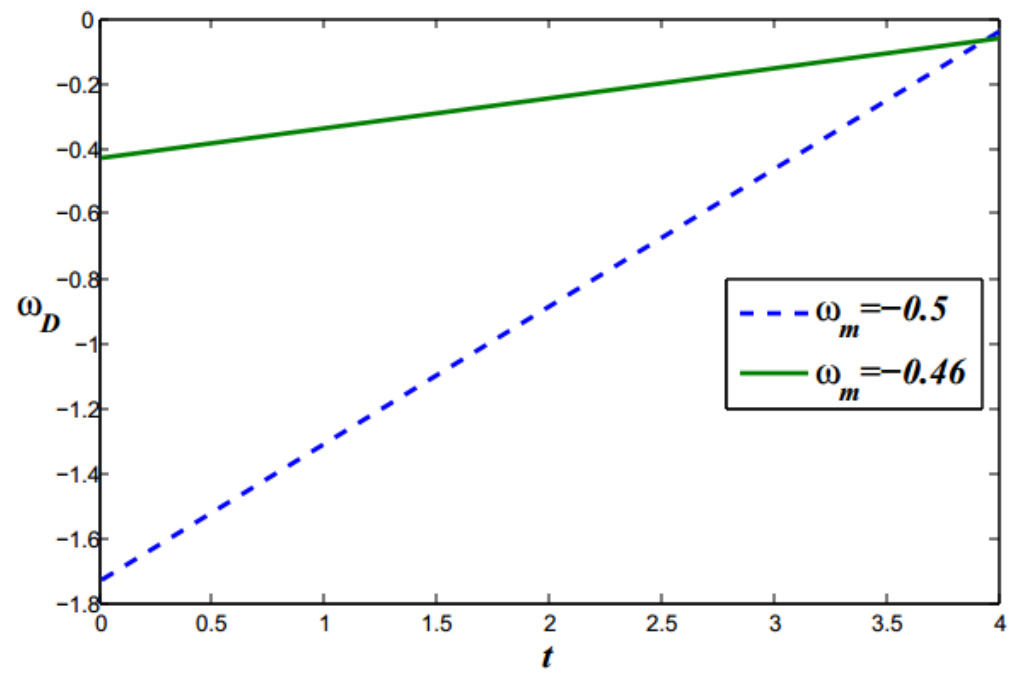

Figure 1. Plot of EoS parameter of dark energy versus time in non-interacting case

Figure 1 describes the behavior of EoS parameter of dark energy versus time. It is observed that the EoS parameter is an increasing function of time and varying in phantom region $\left(-3<\omega_{D}<-1\right)$ and Quintessence region $\left(\omega_{D}>-1\right)$. The rapid increase in early stage of universe depends on the EoS parameter of ordinary matter $\omega_{m}$. Also, it is in good agreement with the theoretical results as well as the recent observations.

\section{Interacting two-fluid model}

Secondly, we consider the interaction between dark energy and barotropic fluids. For this purpose we can write the continuity equations for dark fluid and barotropic fluids as

$$
\dot{\rho}_{m}+3 H\left(\rho_{m}+p_{m}\right)=Q
$$

and

$$
\dot{\rho}_{D}+3 H\left(\rho_{D}+p_{D}\right)=-Q
$$

The quantity $\mathrm{Q}$ expresses the interaction between the dark energy components. Since we are interested in an energy transfer from the dark energy to dark matter, we consider $Q>0$ which ensures that the second law of thermodynamics is fulfilled (Pavon and Wang [49]). Here we emphasize that the continuity equations (33) and (34) imply that the interaction term (Q) should be proportional to a quantity with units of inverse of time, i.e. $Q \propto \frac{1}{t}$. Therefore, a first and natural candidate can be the Hubble factor $H$ multiplied with the energy density. Following Amendola et al. [50] and Guo et al. [51], we consider 
where $\sigma$ is a coupling constant.

$$
Q=3 H \sigma \rho_{m}
$$

Using equation (35) in equation (33) and after integrating, we obtain

$$
8 \pi \rho_{m}=8 \pi \rho_{0}\left[\sin \left(k_{2} t+k_{3}\right)\right]^{-(n+2)\left(1+\omega_{m}-\sigma\right)}
$$

From equations (16), (21) - (23) and (36), we get energy density for dark energy as

$$
\begin{aligned}
8 \pi \rho_{D} & =k_{2}^{2}\left(k_{4} \cos k_{6} t+k_{5} \sin k_{6} t\right)\left\{\frac{1}{k_{1}^{2}}+\left(1+2 n+\frac{1}{k_{1}^{2}}\right) \cot ^{2}\left(k_{2} t+k_{3}\right)\right\} \\
& +k_{6}\left(-k_{4} \sin k_{6} t+k_{5} \cos k_{6} t\right)\left\{(n+2) k_{2} \cot \left(k_{2} t+k_{3} t\right)-\frac{\omega}{2} k_{6}\left(\frac{-k_{4}+k_{5} \cot k_{6} t}{k_{5}+k_{4} \cot k_{6} t}\right)\right\} \\
& -8 \pi \rho_{0}\left[\sin \left(k_{2} t+k_{3}\right)\right]^{-(n+2)\left(1+\omega_{m}-\sigma\right)}
\end{aligned}
$$

From equations (25) and (37), we get

$$
8 \pi p_{m}=8 \pi \omega_{m} \rho_{0}\left[\sin \left(k_{2} t+k_{3}\right)\right]^{-(n+2)\left(1+\omega_{m}-\sigma\right)}
$$

From equations (14), (21) - (23) and (38) we get dark energy pressure as

$$
\begin{aligned}
& 8 \pi p_{D}=\left(k_{4} \cos k_{6} t+k_{5} \sin k_{6} t\right)\left\{k_{6}^{2}-k_{2}^{2}\left(n^{2} \cot ^{2}\left(k_{2} t+k_{3} t\right)-n-1\right)\right\} \\
& +k_{6}\left(k_{4} \sin k_{6} t-k_{5} \cos k_{6} t\right)\left\{(n+1) k_{2} \cot \left(k_{2} t+k_{3} t\right)+\frac{\omega}{2} k_{6}\left(\frac{-k_{4}+k_{5} \cot k_{6} t}{k_{5}+k_{4} \cot k_{6} t}\right)\right\} \\
& -8 \pi \rho_{0} \omega_{m}\left[\sin \left(k_{2} t+k_{3}\right)\right]^{-(n+2)\left(1+\omega_{m}-\sigma\right)}
\end{aligned}
$$

From equations (25), (37) and (39), we get EoS parameter for dark energy as

$$
\begin{aligned}
& \left(k_{4} \cos _{6} t+k_{5} \sin k_{6} t\right)\left\{k_{6}^{2}-k_{2}^{2}\left(n^{2} \cot ^{2}\left(k_{2} t+k_{3} t\right)-n-1\right)\right\}-8 \pi \rho_{0} \omega_{m}\left[\sin \left(k_{2} t+k_{3}\right)\right]^{-(n+2)\left(1+\omega_{m}-\sigma\right)} \\
\omega_{D}= & +k_{6}\left(k_{4} \sin k_{6} t-k_{5} \cos k_{6} t\right)\left\{(n+1) k_{2} \cot \left(k_{2} t+k_{3} t\right)+\frac{\omega}{2} k_{6}\left(\frac{-k_{4}+k_{5} \cot k_{6} t}{k_{5}+k_{4} \cot k_{6} t}\right)\right\} \\
& k_{2}^{2}\left(k_{4} \cos k_{6} t+k_{5} \sin k_{6} t\right)\left\{\cot ^{2}\left(k_{2} t+k_{3} t\right)\left(1+2 n+\frac{1}{k_{1}^{2}}\right)+\frac{1}{k_{1}^{2}}\right\}-8 \pi \rho_{0}\left[\sin \left(k_{2} t+k_{3}\right)\right]^{-(n+2)\left(1+\omega_{m}-\sigma\right)} \\
+ & k_{6}\left(-k_{4} \sin k_{6} t+k_{5} \cos k_{6} t\right)\left\{(n+2) k_{2} \cot \left(k_{2} t+k_{3} t\right)-\frac{\omega}{2} k_{6}\left(\frac{-k_{4}+k_{5} \cot k_{6} t}{k_{5}+k_{4} \cot k_{6} t}\right)\right\}
\end{aligned}
$$

Thus the metric (24) together with (23) and (36)-(40) constitutes Kantowski-Sachs interacting twofluid cosmological model in Brans-Dicke scalar tensor theory of gravitation.

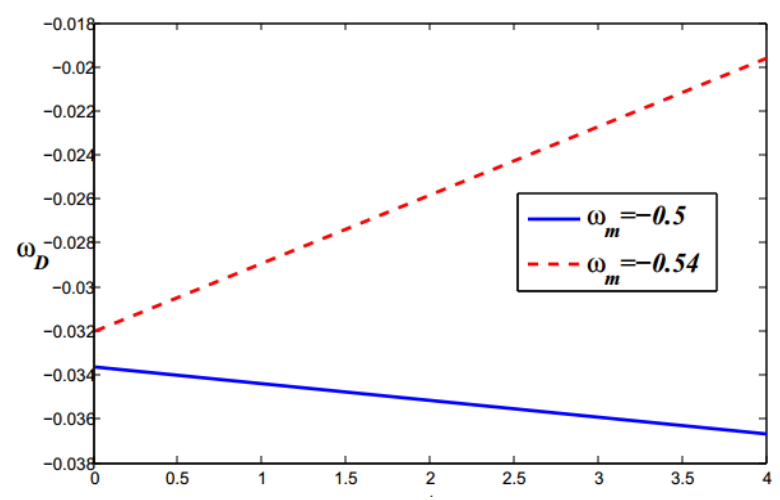

Figure 2. Plot of EoS parameter of dark energy versus time in interacting case 
Figure 2 demonstrates the behavior of the EoS parameter of dark energy versus time. It is observed that the EoS parameter is varying in Quintessence region only.

\section{Some Other Important Properties of the Models}

The spatial volume for the models is

$$
V=(-g)^{\frac{1}{2}}=\left(\frac{k_{1}}{k_{2}} \sin \left(k_{2} t+k_{3}\right)\right)^{n+2} \sin \theta
$$

The average scale factor for the model is

$$
a(t)=V^{\frac{1}{3}}=\left(\frac{k_{1}}{k_{2}} \sin \left(k_{2} t+k_{3}\right)\right)^{\frac{n+2}{3}} \sin ^{1 / 3} \theta
$$

The expansion scalar $\theta$ is given by

$$
\theta=u_{, i}^{i}=k_{2}(n+2) \cot \left(k_{2} t+k_{3}\right)
$$

The shear scalar is given by

$$
\sigma^{2}=\frac{1}{2} \sigma^{i j} \sigma_{i j}=\frac{1}{2}\left(k_{2}(n+2) \cot \left(k_{2} t+k_{3}\right)\right)^{2}
$$

The deceleration parameter $q$ is given by

$$
q=\left(-3 \theta^{-2}\right)\left(\theta_{, i} u^{i}+\frac{1}{3} \theta^{2}\right)=-1+\frac{3}{n+2} \sec ^{2}\left(k_{2} t+k_{3}\right)
$$

The deceleration parameter appearing with negative sign implies accelerating expansion of the universe, which is consistent with the present day observations.

The Hubble's parameter $H$ is given by

$$
H=\frac{1}{3} k_{2}(n+2) \cot \left(k_{2} t+k_{3}\right)
$$

The mean anisotropy parameter $A_{m}$ is given by

$$
A_{m}=2\left(\frac{n-1}{n+2}\right)^{2}
$$

The tensor of rotation $w_{i j}=u_{i, j}-u_{j, i}$ is identically zero and hence this universe is non- rotational.

\section{Conclusions}

In this paper, we have obtained and presented spatially homogeneous Kantowski-Sachs cosmological models filled with barotropic fluid and dark energy in a scalar tensor theory of gravitation proposed by Brans and Dicke [32]. The role of two-fluid either minimally or directly coupled in the evolution of the dark energy parameter has been investigated.

The following are the observations and conclusions:

- The obtained models have singularity at $t=t^{*}$ where $t^{*}=-k_{3} / k_{2}$.

- The volume of the models vanishes at $t=t^{*}$ and the expansion scalar tends to infinity, which shows that the universe starts evolving with zero volume at $t=t^{*}$ with an infinite rate of expansion.

- The EoS parameter of the non-interacting two-fluid model provided by (32) may accommodate the acceptable range $-1.67<\omega_{D}<-0.62$ of SNe Ia data (Knop et al. [7]). While the EoS parameter of the interacting model is varying in only quintessence $\left(\omega_{D}<-1\right)$.

- From equation (45), it is observed that deceleration parameter is negative for small values of $t$ and hence the obtained models represent accelerated expansion of the universe at initial epoch. Recent observations of type Ia supernovae (Perlmutter et al. [6]; Riess et al. [1]) reveal that the 
present universe is in accelerating phase and deceleration parameter lies somewhere in the range $-1<q \leq 0$. It follows that our two-fluid dark energy models of the universe are consistent with the recent observations.

- From (47), one can observe that $A_{m} \neq 0$ and this indicates that these models are anisotropic.

The two models presented here are anisotropic, non-rotating, shearing and also accelerating. Hence they represent not only the early stage of evolution but also the present universe.

\section{References}

1. A. G. Reiss, et al., "Observational evidence from supernovae for an accelerating universe and a cosmological constant", Astron. J. 116, 1009-1038 (1998).

2. A.G. Reiss, et al., "The case for an accelerating universe from supernovae", Publ. Astron. Soc. Pac. 114, 1284-1299 (2000).

3. A.G. Reiss, et al., "Type-Ia supernova discoveries at $z>1$ from the Hubble space telescope: evidence for past deceleration and constraints and dark energy evolution", Astrophys. J. 607, 665-687 (2004).

4. S. Perlmutter, et al. "Measurements1 Of The Cosmological Parameters $\Omega$ and $\Lambda$ " From The First Seven Supernovae at $z \geq 0.35$ ", Astrophys. J. 483, 565-581 (1997).

5. S. Perlmutter, et al. "Discovery of a supernova explosion at half the age of the Universe", Nature 391, 51-54 (1998).

6. S.Perlmutter, et al., "Measurements of Omega and Lambda from 42 High-Redshift Supernovae", Astrophys. J. 517, 565-586 (1999).

7. R. K. Knop, et al. (Supernova Cosmology Project Collaboration) "New Constraints On $\Omega_{M} \Omega_{\Omega}$ and $w$ From an Independent Set of 11 High-Redshift Supernovae Observed With The Hubble Space Telescope", Astrophys. J. 598, 102-137 (2003)

8. R. R. Caldwell, "A Phantom Menace? Cosmological consequences of a dark energy component with super-negative equation of state", Phys. Lett. B 545, 23-29 (2002).

9. Huange, et al., "Holographic explanation of wide-angle power correlation suppression in the cosmic microwave background radiation", J. Cosmol. Astropart. Phys., 05, 013 (2006).

10. Daniel, et al., "Large scale structure as a probe of gravitational slip", Phys. Rev. D, 77, 103513 (2008).

11. O. Akarsu, C. B. Kilinc, "LRS Bianchi type I models with anisotropic dark energy and constant deceleration parameter", Gen. Relativ. Gravit. 42, 119-140 (2010).

12. O. Akarsu, C. B. Kilinc, "Bianchi type III models with anisotropic dark energy", Gen. Relativ. Gravit. 42, 763-775 (2010b).

13. A.K.Yadav, "Some anisotropic dark energy models in Bianchi type-V space-time", Astrophys. Space Sci. 335, 565-575 (2011).

14. A. K. Yadav, L. Yadav, "Bianchi type-III anisotropic dark energy models with constant deceleration parameter", Int. J. Theor. Phys. 50, 218-227 (2011).

15. A. Pradhan, et al., "Bianchi Type-I anisotropic dark energy model with constant deceleration parameter", Int. J. Theor. Phys. 50, 2923-2938 (2011a).

16. A. Pradhan, et al., "A new class of LRS Bianchi type-II dark energy models with variable EoS parameter", Astrophys. Space Sci. 334, 249-260 (2011b).

17. A. Pradhan, A., H. Amirhashchi, B. Saha, "An interacting and non-interacting two-fluid scenario for dark energy in FRW universe with constant deceleration parameter", Astrophys. Space Sci. 333, 343-350 (2011).

18. A. K. Yadav, et al., "Dark Energy Models with Variable Equation of State Parameter", Int. J. Theor. Phys. 50, 871-881 (2011).

19. C.L Bennett, et al., "First year wilkinson microwave anisotropy probe observations: prelimi nary maps and basic results", Astrophys. J. Suppl. Ser. 148, 1-27 (2003).

20. D.N.Spergel, et al. (WMAP Collaboration), "First Year Wilkinson Microwave Anisotropy Probe (WMAP) Observations: Determination of Cosmological Parameters", Astrophys. J. Suppl. Ser.148, 175-194 (2003).

21. M.Tegmark, et al. (SDSS Collaboration), "Cosmological parameters from SDSS and WMAP" Phys. Rev. D 69, 103501 (2004). 
22. R. C. Tolman, "Relativity, thermodynamics, and cosmology. Oxford", Clarendon Press, London, Section 165 (1934).

23. W. Davidson, "The Cosmological Implications of the Recent Counts of Radio Sources: II. An Evolutionary Model", Mon. Not. R. Astron. Soc. 124, 79-93 (1962).

24. M. Cataldo, P. Mella, P. Minning, J. Saavedra, "Interacting cosmic fluids in power-law FriedmannRobertson-Walker cosmological models", Phys. Lett. B, 662, 314-322 (2008).

25. A. Pradhan, H. Amirhashchi, "Dark energy models in anisotropic Bianchi type-III space-time with variable EoS parameter", Astrophys. Space Sci. 332, 441-448 (2011).

26. K.S. Adhav, M.V. Dawande, S. M.Borikar, "Interacting Cosmic Fluids in LRS Bianchi Type-I Cosmological Models", Bulg. Jour. Phys. 38, 371-379 (2011).

27. B. Saha, H. Amirhashchi, A. Pradhan, "Two-fluid scenario for dark energy models in an FRW universe-revisited", Astrophys. Space Sci. 342, 257-267 (2012).

28. H. Amirhashchi, A. Pradhan, B. Saha, "An interacting two-fluid scenario for dark energy in an FRW universe", Chin. Phys. Lett. 28, 039801 (2011).

29. K. S. Adhav, M. V. Dawande, S. M. Borikar, "Kaluza-Klein interacting cosmic fluid cosmological model", Jour. of Theo. and Applied Phys. 6, 33 (2012).

30. D. R. K. Reddy, R. Santhi Kumar, "Two fluid scenario for dark energy model in a scalar-tensor theory of gravitation", Int. J. Theor. Phys. 52, 1362-1369 (2013).

31. H. Amirhashchi, A. Pradhan, Hishamuddin Zainuddin, "Interacting two-fluid viscous dark energy models in a non-flat universe", Research in Astron. Astrophysics 13, 129-138 (2013).

32. C. H. Brans, R. H. Dicke, "Mach's principle and a relativistic theory of gravitation", Phys.Rev.124, 925-935(1961).

33. T. Singh, L. N. Rai, "Scalar-tensor theories of gravitation: Foundations and prospects" Gen.Rel.Grav 15, 875- 902 (1983).

34. D. R. K.Reddy, V. U. M.Rao, "Field of a charged particle in Brans-Dicke theory of gravitation", J. Phys. A: Math. Gen., 14, 1973-1976 (1981).

35. A. Banerjee, N. O. Santos, "Bianchi type-II cosmological models in Brans-Dicke theory", Nuovo Cimento, 67B, 31-40 (1982).

36. Shri Ram." Spatially homogeneous and anisotropic cosmological solution in Brans-Dicke theory", Gen. Relativ. Gravit., 15, 635-640 (1983).

37. Shri Ram, D. K Singh, "Exact Bianchi type $\mathrm{VI}_{0}$ cosmological solutions with matter in Brans-Dicke theory", Astrophys. Space Sci., 103, 21-26 (1984).

38. M. S. Berman, et al." Brans-Dicke static universes", Gen. Relativ. Gravit. 21, 287-292 (1989).

39. D. R. K. Reddy," A String cosmological model in Brans-Dicke theory of gravitation", Astrophys. Space Sci., 286, 365-371 (2003).

40. D. R. K. Reddy, R. L. Naidu, "Five dimensional string cosmological models in a scalar-tensor theory of gravitation", Astrophys. Space Sci., 307, 395-398 (2007).

41. K.S. Adhav, A. S. Nimkar, M. R. Ugale, M.V. Dawande, "N-dimensional string cosmological model in Brans-Dicke theory of gravitation", Astrophys. Space Sci., 310, 231-235 (2007).

42. V. U. M. Rao, M. Vijaya Santhi, "Bianchi Type-II, VIII \& IX Perfect fluid cosmological models in Brans Dicke theory of gravitation", J. Mod. Phys., 2, 1222-1228 (2011).

43. V.U.M.Rao, M. Vijaya Santhi, "Bianchi type-II, VIII \& IX perfect fluid magnetized cosmological models in Brans-Dicke theory of gravitation", Astrophys. Space Sci., 337, 387-392 (2012).

44. V.U.M.Rao, M. Vijaya Santhi, "Bianchi Types II, VIII, and IX String cosmological models in BransDicke theory of gravitation", ISRN Math. Phys., doi:10.5402/2012/573967 (2012).

45. R. L. Naidu, K. Dasu Naidu, K. Shobhan Babu, D.R.K. Reddy, "A five dimensional Kaluza-Klein bulk viscous string cosmological model in Brans-Dicke scalar-tensor theory of gravitation", Astrophys. Space Sci., 347, 197-201 (2013).

46. T. Vidya Sagar, et al., "A five dimensional Kaluza-Klein bulk viscous string cosmological model in Brans-Dicke scalar-tensor theory of gravitation", Astrophys. Space Sci., 349, 479-483 (2014).

47. S. Das, A.M. Abdulla, "An Interacting Model of Dark Energy in Brans-Dicke Theory", Astrophys. Space Sci.,351, 651-660 (2014).

48. D. D.Pawar, Y.S. Solanke, "Exact Kantowski-Sachs Anisotropic Dark Energy Cosmological Models in Brans Dicke Theory of Gravitation", Int. J. Theor. Phys., 53, 3052-3065 (2014). 
49. D. Pavon, B. Wang, "Le Chatelier-Braun principle in cosmological physics", Gen. Relativ. Gravit. 41, 1-5(2009).

50. L. Amendola, G. Camargo Campos, R. Rosenfeld, "Consequences of dark matter-dark energy interaction on cosmological parameters derived from type Ia supernova data", Phys. Rev. D 75, 083506 (2007).

51. Z. K. Guo, N. Ohta, S. Tsujikawa, "Probing the coupling between dark components of the universe", Phys. Rev.D, 76, 023508 (2007). 